\title{
A GENERALIZATION OF SLOWLY VARYING FUNCTIONS
}

\author{
D. DRASIN ${ }^{1}$ AND E. SENETA
}

ABSTRACT. This note establishes that if the main part of the definition of a slowly varying function is relaxed to the requirement that $\limsup _{x \rightarrow \infty} \psi(\lambda x) / \psi(x)<\beta<$ $\infty$ for each $\lambda>0$, then $\psi(x)=L(x) \theta(x)$, where $L$ is slowly varying and $\theta$ is bounded. This is done by obtaining a representation for the function $\psi$.

1. Introduction. We are concerned with a function $\psi$ which is positive, finite, measurable and defined on $[A, \infty)$ for some $A>0$, and such that

$$
\limsup _{x \rightarrow \infty} \frac{\psi(\lambda x)}{\psi(x)}<\beta<\infty
$$

for each $\lambda>0$, where $\beta(\geqslant 1)$ is independent of $\lambda>0$. We shall call such a function $S-O$ varying. Notice that this definition of an $S-O$ varying function differs from that in [4, Appendix]; the reason for the redefinition will be made clear shortly.

Condition (1) generalizes the notion of a slowly varying function [4], which requires that $\lim _{x \rightarrow \infty} \psi(\lambda x) / \psi(x)=1$; our development elucidates the structure of $S-O$ varying $\psi$ in relation to the properties of slowly varying functions.

Notice that (1) implies that

$$
0<\frac{1}{\beta}<\liminf _{x \rightarrow \infty} \frac{\psi(\lambda x)}{\psi(x)}
$$

for each $\lambda>0$. Passing to the usual additive reformulation, put $f(x)=\log \psi\left(e^{x}\right)$. Then (1) and (2) imply that

$$
\forall u \in(-\infty, \infty), \quad \limsup _{x \rightarrow \infty}|f(x+u)-f(x)| \leqslant K<\infty,
$$

where $K$ can be taken as $\log \beta$. The main purpose of this note is to prove

Theorem. Suppose $f$ is defined, finite and measurable on $[B, \infty)$ for some finite $B$. Then $f$ satisfies (3) for some $K$ if and only if $f$ has the representation

$$
f(x)=a(x)+\int_{n_{0}}^{x} \varepsilon(u) d u
$$

Received by the editors January 3, 1985 and, in revised form, April 1, 1985.

1980 Mathematics Subject Classification. Primary 26A12.

Key words and phrases. Slowly varying function, uniform convergence, $R-O$ varying, representation.

${ }^{1}$ Work done while this author was visiting Imperial College, London. Research supported in part by the National Science Foundation. 
for all $x \geqslant n_{0}$, where $n_{0}(\geqslant B)$ is some fixed number, $a(x)$ is measurable and bounded on $\left[n_{0}, \infty\right)$, and $\varepsilon(x)$ is measurable on $\left[n_{0}, \infty\right)$, with $\varepsilon(x) \rightarrow 0$ as $x \rightarrow \infty$.

For a slowly varying function in its additive version, $K$ in (3) is zero. A bounded $f$ satisfying (3), where $K$ cannot be taken as zero, is $f(x)=(-1)^{[x]}$. An example of an unbounded $f$ of this kind can be obtained by adding the additive version of any unbounded slowly varying function; e.g., $f(x)=(-1)^{[x]}+\log x$.

To place our result within the context of existing theory of regular variation and its generalizations, we return from the additive formulation to consider all measurable functions $\psi$ which for $x \geqslant A_{1}$ can be represented in the form

$$
\psi(x)=\exp \left\{\alpha(x)+\int_{A_{1}}^{x} \beta(t) t^{-1} d t\right\},
$$

where $\alpha(x)$ and $\beta(x)$ are measurable. We then have the following correspondences, where the Theorem corresponds to IV:

I. $\psi$ slowly varying [4] $\Leftrightarrow \alpha(x) \rightarrow \alpha, \beta(x) \rightarrow 0$.

II. $\psi R-O$ varying [4, Appendix] $\Leftrightarrow \alpha(x)$ bounded, $\beta(x)$ bounded.

III. $\psi E R$ varying $[2, \S 2 ; 3,(3.8)] \Leftrightarrow \alpha(x) \rightarrow \alpha, \beta(x)$ bounded.

IV. $\psi S-O$ varying $\Leftrightarrow \alpha(x)$ bounded, $\beta(x) \rightarrow 0$.

It is clear that IV is a subclass of II, the $S-O$ terminology being justified because of the behaviour of $\beta(x)$. The example of an $S-O$ varying function mentioned earlier shows that IV is not a subclass of III. Finally, a positive measurable function $\psi$ is $S-O$ varying on $[A, \infty)$ if and only if it can be written in the form $\psi(x)=L(x) \theta(x), x \geqslant A$, where $L$ is slowly varying at infinity and $\theta(x)$ is such that $\theta(x)$ and $1 / \theta(x)$ are bounded on $[A, \infty)$. For further relaxation of condition (1) see $[1 ; 2$, Corollary 3.4$]$.

\section{Results.}

LEMMA. Under the conditions of the Theorem and integer $U$,

$$
\sup _{U>0} \limsup _{x \rightarrow \infty}\left\{\sup _{0 \leqslant u \leqslant U}|f(x+u)-f(x)|\right\}<\infty .
$$

Proof. By [1, Theorem 1], (3) implies that for any compact interval $I$ in $(-\infty, \infty)$, $-\infty<\liminf _{x \rightarrow \infty}\left\{\inf _{u \in I}(f(x+u)-f(x))\right\}, \quad \limsup _{x \rightarrow \infty}\left\{\sup _{u \in I}(f(x+u)-f(x))\right\}<\infty$,

whence

$$
\limsup _{x \rightarrow \infty}\left\{\sup _{u \in I}|f(x+u)-f(x)|\right\}<\infty .
$$

Now let $C / 2$ exceed both $\sup _{u \geqslant 0} \lim \sup _{x \rightarrow \infty}|f(x+u)-f(x)|$ (from (3)), and $\limsup _{x \rightarrow \infty} \sup _{0 \leqslant u \leqslant 1}|f(x+u)-f(x)|$ (from (5)). Then for any positive integer $U$ there exists $X=X(U)>0$ such that for all $x \geqslant X$,

$$
\begin{gathered}
|f(x+k)-f(x)|<C / 2 \quad(k=1,2, \ldots, U), \\
\sup _{0 \leqslant u \leqslant 1}|f(x+u)-f(x)|<C / 2 .
\end{gathered}
$$


Then for $0 \leqslant u \leqslant U$ and $x \geqslant X$,

$$
|f(x+u)-f(x)| \leqslant|f(x+[u])-f(x)|+|f(x+u)-f(x+[u])|<C .
$$

Proof of The Theorem. Define $n_{0}<n_{1}<n_{2}<\cdots$ as follows. Let $n_{0} \geqslant X(1)$ be an integer. Having defined $n_{k-1}$, (4) allows us to find a positive integer $m_{k}$ such that, on setting $n_{k}=n_{k-1}+k\left(m_{k}+1\right)$,

$$
\sup _{x \geqslant n_{k}} \sup _{0 \leqslant u \leqslant k+1}|f(x+u)-f(x)|<C .
$$

Now for each $k=1,2, \ldots$ and $m=0,1, \ldots, m_{k}$ define

$$
\begin{aligned}
\varepsilon(x)= & \left(f\left(n_{k-1}+m k+k\right)-f\left(n_{k-1}+m k\right)\right) / k, \\
& n_{k-1}+m k \leqslant x<n_{k-1}+m k+k .
\end{aligned}
$$

On this interval $|\varepsilon(x)| \leqslant C / k$ so $\varepsilon(x) \rightarrow 0$ as $x \rightarrow \infty$.

Now, given any $x \geqslant n_{0}$, we may write it as $x=n_{k-1}+m k+y$ for some $k \in\{1,2, \ldots\}, m \in\left\{0, \ldots, m_{k}\right\}$ and $0 \leqslant y<k$, so that

$$
\begin{aligned}
f(x)-f\left(n_{0}\right)-\int_{n_{0}}^{x} \varepsilon(u) d u= & f(x)-f\left(n_{k-1}+m k\right)-\int_{n_{k-1}+m k}^{x} \varepsilon(u) d u \\
= & f\left(n_{k-1}+m k+y\right)-f\left(n_{k-1}+m k\right) \\
& -\frac{y}{k}\left(f\left(n_{k-1}+m k+k\right)-f\left(n_{k-1}+m_{k}\right)\right),
\end{aligned}
$$

which by (6) is in absolute value $<2 C$.

AcKNOWLEDGEMENTS. The problem of establishing the factorization $\psi(x)=$ $L(x) \theta(x)$ was put to one of the authors (E.S.) by Dr. R. Vasudeva, University of Mysore. Our presentation has been greatly improved by suggestions from the referee.

\section{REFERENCES}

1. S. Aljancić and D. Arandelović, O-regularly varying functions, Publ. Inst. Math. Acad. Serbe Sci. 22 (1977), 5-22.

2. N. H. Bingham and C. M. Goldie, Extensions of regular variation. I: Uniformity and quantifiers, Proc. London Math. Soc. 44 (1982), 473-496.

3. (1982), 497-534. , Extensions of regular variation. II: Representations and indices, Proc. London Math. Soc. 44

4. E. Seneta, Regularly varying functions, Lecture Notes in Math., vol. 508, Springer-Verlag, Berlin, 1976.

Department of Mathematics, Purdue University, West Lafayette, Indiana 47907

Department of Mathematical Statistics, University of Sydney, New South Wales 2006 , Australia 